\title{
ON THE EXISTENCE, REGULARITY AND LARGE TIME BEHAVIOR OF A TWO-DIMENSIONAL PARABOLIC-ELLIPTIC EDDY CURRENT PROBLEM
}

\author{
$\mathrm{BY}$
}

\section{A. ALDROUBI}

National Institutes of Health, Bethesda, Maryland

\begin{abstract}
We consider a two-dimensional time-dependent eddy current model formulated as a parabolic-elliptic interface problem. Existence of solutions is established using Laplace transform techniques, potential theory and integral equations methods. Regularity results are shown, from which the existence of classical solutions follow. A large time behavior for the solution is established.
\end{abstract}

1. Introduction. The interface problem we consider, is a model for the scattering of electromagnetic waves due to metallic obstacles: When an electromagnetic field travelling in air encounters a metallic obstacle it generates eddy currents in the metal producing power losses. In turn, the eddy currents in the obstacle distort the incident field. More specifically, we consider a nonperiodic time-dependent eddy current problem corresponding to the interaction between transverse magnetic fields travelling in air and cylindrical obstacles.

Conduction current in air and displacement current in metal are neglected. The obstacle we consider is a nonferromagnetic metal with the permeability of the air and with a constitutive relation described by the standard Ohm's law.

In the case of a slowly varying incident field, the mathematical formulation of our problem leads to a parabolic-elliptic interface problem in the plane which can be described as follows:

The parabolic-elliptic problem (PEP). Find $u, v$ such that

$$
\begin{gathered}
q \frac{\partial u}{\partial t}=\Delta u \quad \text { in } \Omega \times(0, \infty), \quad \Delta v=0 \quad \text { in } \Omega^{+} \times(0, \infty), \\
u(x, 0)=0 \quad \text { in } \Omega, \\
u^{-}=v^{+}+\phi \quad \text { on } \delta \Omega \times(0, \infty), \quad u_{n}^{-}=v_{n}^{+}+\psi \quad \text { on } \delta \Omega \times(0, \infty), \\
v(x, t)=O(1) \quad \text { as }|x| \rightarrow \infty, \quad \nabla v(x, t)=O\left(|x|^{-2}\right) \quad \text { as }|x| \rightarrow \infty .
\end{gathered}
$$

Received July 17, 1989.

This work was partially supported by the Air Force Office of Scientific Research under grants AFOSR-860085, AFSOR-85-0307, and by the National Science Foundation.

This work was completed while the author was at the Dept. of Math., Virginia Polytechnic Institute and State University. 
Here $\Omega \subset \mathbb{R}^{2}$ is the interior region of a smooth Jordan curve $\delta \Omega$ and $\Omega^{+}$is the exterior region; $q$ is a suitably smooth, strictly positive function on $\bar{\Omega}, \phi$ and $\psi$ are prescribed functions on $\delta \Omega \times[0, \infty)$. The subscript $n$ indicates differentiation in the outer normal direction to $\delta \Omega$, and $f^{-}\left(f^{+}\right)$denotes the limit on $\delta \Omega \times(0, \infty)$ of a function $f$ defined on $\Omega \times(0, \infty)\left(f\right.$ defined on $\left.\Omega^{+} \times(0, \infty)\right)$.

Problem (PEP) is a limit case corresponding to an exterior incident field having low frequency components. This formulation gives a good description of the physical phenomenon near and inside the obstacle but it is expected to be inaccurate far away from the obstacle. A more accurate model in the exterior domain is obtained by replacing the Laplace equation by the wave equation.

The Mathematical formulation of the time-dependent eddy current problem (PEP) was introduced and studied by R. C. MacCamy and M. Suri [4]. There, the authors use variational methods to prove the existence of a generalized solution. However, they do not obtain regularity results.

This paper is about existence, regularity, and large time behavior of the solutions of (PEP). Our method involves Laplace transform techniques, potential theory, and integral equations methods. We use the Laplace transform to reduce (PEP) into an elliptic-elliptic interface problem. We solve the reduced problem and get estimates for the solution. We use these estimates to invert the Laplace transform and to find a solution to Problem (PEP). In Sec. 6, for completeness, we have included a brief discussion about the derivation of (PEP) from physical principles.

In connection with our work, a time periodic version of the two-dimensional eddy current problem is treated by S. I. Hariharan and R C. MacCamy [1,2]. There, the authors treat two cases: The limit case of low frequency approximation resulting in Laplace equation for the exterior region and the case where no low frequency approximation is assumed leading to a Helmholtz equation.

2. Statements of the results. We introduce the following definitions. Let $\mathbf{X}$ be a separable Hilbert space, $m$ a nonnegative integer and $\rho$ a real number; $Z^{m}[\mathbf{X}, \rho]$ will denote a space of smooth functions from $(0, \infty)$ into $\mathbf{X}$ satisfying a growth condition:

$$
\begin{aligned}
Z^{m}[\mathbf{X}, \rho]:= & \left\{\varphi \in C^{m}[[0, \infty): x]: \varphi^{(h)}(0)=0 \text { for } 0 \leq h \leq m-1\right], \\
& \left.\int_{0}^{\infty}\left\|\varphi^{(h)}(t)\right\| e^{-\rho t} d t<\infty \text { for } 0 \leq h \leq m\right\} .
\end{aligned}
$$

Let $\Omega$ be as before and $R$ be any open bounded domain in $\mathbb{R}^{2}$ with smooth boundary $\delta R$ such that $\Omega \subset R$. We define $\Omega_{R}^{+}$and $\Omega_{R}^{+}(\delta)$ by

$$
\begin{gathered}
\Omega_{R}^{+}=\left\{x \in \mathbb{R}^{2}: x \in \Omega^{+} \cap R\right\}, \\
\Omega_{R}^{+}(\delta)=\left\{x \in \Omega_{R}^{+}: \operatorname{dist}(x, \delta \Omega)>\delta, \delta>0\right\} .
\end{gathered}
$$

Our first result is an existence Theorem for (PEP). For simplicity, we will require throughout the paper that $\delta \Omega$ is $C^{\infty}$ and that the function $q$ in $(1.1)$ is $C^{\infty}(\bar{\Omega})$. 
THEOREM 2.1. Assume that $\phi \in Z^{I[m / 2]+3}\left[H^{m+3 / 2}(\delta \Omega), \rho_{0}\right], \quad \psi \in Z^{I[m / 2]+3}$ $\left[H^{m+1 / 2}(\delta \Omega), \rho_{1}\right]$, for some integer $m \geq 0$ and some real numbers $\rho_{0}, \rho_{1}$. There exists a unique solution $(u, v)$ of (PEP) with

$$
\begin{gathered}
u \in C\left[[0, \infty): H^{m+2}(\Omega)\right] \cap C^{1}\left[[0, \infty): H^{m}(\Omega)\right], \\
v \in C\left[[0, \infty): H^{m+2}\left(\Omega_{R}^{+}\right)\right] \cap C\left[[0, \infty): C^{1}\left(\Omega_{R}^{+}(\delta)\right)\right] .
\end{gathered}
$$

Here $I[r]$ is the smallest integer greater than or equal to $r$.

REMARKS.

1. The implicit requirement that $\phi$ and $\psi$ and some of their derivatives vanish at $t=0$, is a compatibility condition with the zero initial condition for $u$.

2. Using Theorem 2.1 and the Sobolev imbedding Theorems, we can obtain classical solutions of (PEP) as long as the data $\phi$ and $\psi$ are sufficiently smooth.

Our next Theorem is a large time behavior of the solutions of (PEP). The result is that if $\phi(t)$ and $\psi(t)$ decay sufficiently fast then the solution will tend to a constant as $t \rightarrow \infty$.

THEOREM 2.2. Assume that $\phi \in Z^{I[m / 2]+3}\left[H^{m+3 / 2}(\delta \Omega), \rho_{0}\right], \quad \psi \in Z^{I[m / 2]+3}$ $\left[H^{m+1 / 2}(\delta \Omega), \rho_{1}\right]$ for some integer $m \geq 0, \rho_{0}<0, \rho_{1}<0$. If $(u, v)$ is a solution of (PEP) then $\exists c^{1}, c^{2}\left(\Omega_{R}^{+}\right), r_{0}>0$ such that

$$
\begin{aligned}
& \text { (i) }\left\|u-A^{-1} \int_{0}^{t} \int_{\partial \Omega} \psi\right\|_{m+2, \Omega} \leq c^{1} e^{-r_{0} t}, \\
& \text { (ii) }\left\|v-A^{-1} \int_{0}^{t} \int_{\partial \Omega} \psi\right\|_{m+2, \Omega_{R}^{+}} \leq c^{2} e^{-r_{0} t},
\end{aligned}
$$

with $A=\int_{\Omega} q$.

\section{REMARKS.}

1. The assumption $\rho_{0}<0, \rho_{1}<0$ in the statement of the Theorem is a statement about the rate of decay of $\phi(t)$ and $\psi(t)$ as $t \rightarrow \infty$.

2. The condition $\rho_{1}<0$ implies that $\lim _{t \rightarrow \infty} \int_{0}^{t} \int_{\partial \Omega} \psi$ exists. This implies that the solution of (PEP) converges to a constant as $t \rightarrow \infty$ and that the convergence rate is exponential. Moreover, this constant is independent of the function $\phi$.

3. The reduced and degenerate eddy current problems. Formally, taking the Laplace transform in (1.1) we get an elliptic-elliptic interface problem that we refer to as the reduced eddy current problem (EP):

The elliptic-elliptic problem (EP). Find $\hat{u}, \hat{v}$ such that

$$
\begin{gathered}
\Delta \hat{u}-s q \hat{u}=0 \quad \text { in } \Omega, \quad \Delta \hat{v}=0 \quad \text { in } \Omega^{+}, \\
\hat{u}^{-}=\hat{v}^{+}+\hat{\phi} \quad \text { on } \delta \Omega, \quad \hat{u}_{n}^{-}=\hat{v}_{n}^{+}+\hat{\psi} \quad \text { on } \delta \Omega, \\
\hat{v}=O(1) \quad \text { as }|x| \rightarrow \infty, \quad|\nabla \hat{v}|=O\left(|x|^{-2}\right) \quad \text { as }|x| \rightarrow \infty .
\end{gathered}
$$

REMARK. If we put $s=i \eta$ in (EP), we get the time periodic version of the eddy current problem treated in $[1,2]$. 
Associated with (EP) is another elliptic-elliptic interface problem that we call the degenerate eddy current problem (DEP):

The elliptic-elliptic problem $(D E P)$. Find $\hat{u}, \hat{v}$ such that

$$
\begin{aligned}
& \Delta \hat{u}=f \quad \text { in } \Omega, \quad \Delta \hat{v}=0 \quad \text { in } \Omega^{+}, \\
& \hat{u}^{-}=\hat{v}^{+}+\hat{\phi} \quad \text { on } \delta \Omega, \quad \hat{u}_{n}^{-}=\hat{v}_{n}^{+}+\hat{\psi} \quad \text { on } \delta \Omega, \\
& \hat{v}=O(1) \quad \text { as }|x| \rightarrow \infty, \quad|\nabla \hat{v}|=O\left(|x|^{-2}\right) \quad \text { as }|x| \rightarrow \infty \text {. }
\end{aligned}
$$

Before we proceed, we introduce the simple layer, double layer, and volume potential for the Laplace operator and some of their properties.

$$
\begin{gathered}
\mathscr{S}[\varphi](x)=(2 \pi)^{-1} \int_{\delta \Omega} \varphi(y) \log (|x-y|) d \sigma(y), \\
\mathscr{D}[\varphi](x)=(2 \pi)^{-1} \int_{\delta \Omega} \varphi(y) \partial_{n_{y}}(\log (|x-y|)) d \sigma(y), \\
\mathscr{V}[f](x)=(2 \pi)^{-1} \int_{\Omega} f(y) \log (|x-y|) d y,
\end{gathered}
$$

where $\partial_{n_{y}}$ denotes the derivative with respect to the outer normal of $\delta \Omega$. We have the following technical Lemmas.

Lemma 3.1. Let $m$ be an integer with $m \geq 0$.

(i) The operator $\mathscr{S}$ defines bounded linear operators from $H^{m+1 / 2}(\delta \Omega)$ into $H^{m+2}(\Omega)$, from $H^{m+1 / 2}(\delta \Omega)$ into $H^{m+2}\left(\Omega_{R}^{+}\right)$, and from $H^{m+1 / 2}(\delta \Omega)$ into $C^{n}\left(\Omega_{R}^{+}(\delta)\right), \forall \delta>0, \forall n \in \mathbb{N}$.

(ii) The operator $\mathscr{D}$ defines bounded linear operators from $H^{m+3 / 2}(\delta \Omega)$ into $H^{m+2}(\Omega)$, from $H^{m+3 / 2}(\delta \Omega)$ into $H^{m+2}\left(\Omega_{R}^{+}\right)$, and from $H^{m+3 / 2}(\delta \Omega)$ into $C^{n}\left(\Omega_{R}^{+}(\delta)\right), \forall \delta>0, \forall n \in \mathbb{N}$.

(iii) The operator $\mathscr{V}$ defines bounded linear operators from $H^{m}(\Omega)$ into $H^{m+2}(\Omega)$, from $H^{m}(\Omega)$ into $H^{m+2}\left(\Omega_{R}^{+}\right)$, and from $H^{m}(\Omega)$ into $C^{n}\left(\Omega_{R}^{+}(\delta)\right)$, $\forall \delta>0, \forall n \in \mathbb{N}$.

Moreover,

$\Delta \mathscr{S}[\varphi]=\Delta \mathscr{D}[\psi]=0 \quad$ in $\Omega \cup \Omega^{+}, \quad \Delta \mathscr{V}[f]=f \quad$ in $\Omega, \quad \Delta \mathscr{V}[f]=0 \quad$ in $\Omega^{+}$, $\left(\frac{\partial}{\partial n} \mathscr{S}[\varphi]\right)^{+}-\left(\frac{\partial}{\partial n} \mathscr{S}[\varphi]\right)^{-}=\varphi \quad$ on $\delta \Omega, \quad(\mathscr{S}[\varphi])^{+}-(\mathscr{S}[\varphi])^{-}=0 \quad$ on $\delta \Omega$, $(\mathscr{D}[\varphi])^{+}-(\mathscr{D}[\varphi])^{-}=-\varphi \quad$ on $\delta \Omega, \quad\left(\frac{\partial}{\partial n} \mathscr{D}[\varphi]\right)^{+}-\left(\frac{\partial}{\partial n} \mathscr{D}[\varphi]\right)^{-}=0 \quad$ on $\delta \Omega$, $(\mathscr{V}[f])^{+}-(\mathscr{V}[f])^{-}=0 \quad$ on $\delta \Omega, \quad\left(\frac{\partial}{\partial n} \mathscr{V}[f]\right)^{+}-\left(\frac{\partial}{\partial n} \mathscr{V}[f]\right)^{-}=0 \quad$ on $\delta \Omega$. 
Lemma 3.2. There exists $c, r \in \mathbb{R}$ such that $\forall x \in \mathbb{R}$ with $|x| \geq r$ we have,

(i)

$$
\begin{gathered}
\left|\mathscr{S}[\varphi](x)-\left((2 \pi)^{-1} \int_{\delta \Omega} \varphi\right) \log \right| x|| \leq c|x|^{-1} \int_{\delta \Omega} \varphi \\
\left|\nabla\left(\mathscr{S}[\varphi](x)-\left((2 \pi)^{-1} \int_{\delta \Omega} \varphi\right) \log |x|\right)\right| \leq c|x|^{-2} \int_{\delta \Omega} \varphi,
\end{gathered}
$$

(ii)

(iii)

$$
\begin{gathered}
|\mathscr{D}[\varphi](x)| \leq c|x|^{-1} \int_{\delta \Omega} \varphi, \\
|\nabla \mathscr{D}[\varphi](x)| \leq c|x|^{-2} \int_{\delta \Omega} \varphi,
\end{gathered}
$$

$$
\begin{gathered}
\left|\mathscr{V}[f](x)-\left((2 \pi)^{-1} \int_{\Omega} f\right) \log \right| x|| \leq c|x|^{-1} \int_{\Omega} f \\
\left|\nabla\left(\mathscr{V}[f](x)-\left((2 \pi)^{-1} \int_{\Omega} f\right) \log |x|\right)\right| \leq c|x|^{-2} \int_{\Omega} f .
\end{gathered}
$$

The proofs of Lemmas 3.1 and 3.2 can be found in [3].

Before we state our next Theorem, we make the following definitions. We define $\mathscr{S}^{i}[\varphi], \mathscr{D}^{i}[\varphi], \mathscr{V}^{i}[f]$ to be the restriction of $\mathscr{S}[\varphi], \mathscr{D}[\varphi], \mathscr{V}[f]$ onto $\Omega$, and $\mathscr{S}^{e}[\varphi], \mathscr{D}^{e}[\varphi], \mathscr{V}^{e}[f]$ to be the restriction of $\mathscr{S}[\varphi], \mathscr{D}[\varphi], \mathscr{V}[f]$ onto $\Omega^{+}$.

THEOREM 3.1. If $f \in H^{m}(\Omega), \hat{\phi} \in H^{m+3 / 2}(\delta \Omega), \hat{\psi} \in H^{m+1 / 2}(\delta \Omega)$ for some integer $m \geq 0$, then (DEP) has a solution $(\hat{u}, \hat{v}) \in H^{m+2}(\Omega) \times H^{m+2}\left(\Omega_{R}^{+}\right)$if and only if $f$ and $\hat{\psi}$ are related by the compatibility relation

$$
\int_{\Omega} f=\int_{\delta \Omega} \hat{\psi} \text {. }
$$

Moreover, if (DEP) has a solution, then it has infinitely many solutions that are given by

$$
\begin{aligned}
\hat{u} & =\mathscr{V}^{i}[f]+\mathscr{D}^{i}[\hat{\phi}]-\mathscr{S}^{i}[\hat{\psi}]+c, \\
\hat{v} & =\mathscr{V}^{e}[f]+\mathscr{D}^{e}[\hat{\phi}]-\mathscr{S}^{e}[\hat{\psi}]+c,
\end{aligned}
$$

where $c \in \mathbb{C}$ is an arbitrary constant.

Proof. Assuming $(\hat{u}, \hat{v})$ is a solution of (PEP), we integrate $(3.2)_{1}$ and (3.2) 2 over $\Omega$ and $\Omega^{+}$, respectively, and use integration by parts to get

$$
\begin{gathered}
\int_{\Omega} \Delta \hat{u}=\int_{\delta \Omega} \hat{u}_{n}^{-}=\int_{\Omega} f, \\
\int_{\Omega^{+}} \Delta \hat{v}=-\int_{\delta \Omega} \hat{v}_{n}^{+}=0 .
\end{gathered}
$$

Using (3.5), (3.6) and the transition relation across the boundary $\left(\hat{u}_{n}^{-}=\hat{v}_{n}^{+}+\hat{\psi}\right)$ we conclude that the compatibility relation (3.3) must hold. 
Next, assume that $(\hat{u}, \hat{v})$ is a solution of (DEP) for $(f, \hat{\phi}, \hat{\psi})=(0,0,0)$. We multiply $(3.1)_{1}$ by $\overline{\hat{u}}$, the complex conjugate of $\hat{u}$, and $(3.1)_{2}$ by $\overline{\hat{v}}$. We then integrate by parts to get

$$
\begin{gathered}
\int_{\delta \Omega} \hat{u}_{n}^{-}(\overline{\hat{u}})^{-}-\int_{\Omega}|\nabla \hat{u}|^{2}=0, \\
-\int_{\delta \Omega} \hat{v}_{n}^{+}(\overline{\hat{v}})^{+}-\int_{\Omega^{+}}\left|\nabla \hat{v}_{n}^{+}\right|^{2}=0 .
\end{gathered}
$$

Adding (3.7) and (3.8) we get

$$
\int_{\Omega}|\nabla \hat{u}|^{2}+\int_{\Omega^{+}}|\nabla \hat{v}|^{2}=0
$$

from which it is easy to see that $\hat{u} \equiv c_{1}, \hat{v} \equiv c_{2}$, where $c_{1}, c_{2} \in \mathbb{C}$. Using the transition relation across the boundary $\left(\hat{u}^{-}=\hat{v}^{+}\right)$yields $c_{1}=c_{2}=c$.

It only remains to show that if (3.3) holds then $(\hat{u}, \hat{v})$ given by (3.4) satisfy (DEP). From potential theory we have that

$$
\Delta \mathscr{V}^{i}[f] \equiv f, \quad \Delta \mathscr{D}^{i}[\hat{\phi}] \equiv \mathscr{S}^{i}[\hat{\psi}] \equiv \Delta \mathscr{V}^{e}[f] \equiv \Delta \mathscr{D}^{e}[\hat{\phi}] \equiv \mathscr{S}^{e}[\hat{\psi}] \equiv 0 .
$$

Hence $\Delta \hat{u}=f, \Delta \hat{v}=0$. Using Lemma 3.1 we immediately get that $\hat{u}^{-}=\hat{v}^{+}+\hat{\phi}$ and $\hat{u}_{n}^{-}=\hat{v}_{n}^{+}+\hat{\psi}$. To show that the growth conditions as $|x| \rightarrow \infty$ are satisfied, we rewrite $\hat{v}$ as follows:

$$
\begin{aligned}
\hat{v}= & \left(\mathscr{V}^{e}[f]-\left((2 \pi)^{-1} \int_{\Omega} f\right) \log |x|\right)+\mathscr{D}^{e}[\varphi] \\
& -\left(\mathscr{S}^{e}[\hat{\psi}]-\left((2 \pi)^{-1} \int_{\delta \Omega} \hat{\psi}\right) \log |x|\right) \\
& +c+(2 \pi)^{-1}\left(\left(\int_{\Omega} f-\int_{\delta \Omega} \hat{\psi}\right) \log |x|\right) .
\end{aligned}
$$

Because of the compatibility condition (3.3), the last term of the right side of the equality is zero. Using Lemma 3.2 on the remaining terms shows that the growth conditions as $|x| \rightarrow \infty$ are satisfied.

Our next Theorem gives an equivalent integral equation formulation of the reduced eddy current problem (EP).

THEOREM 3.2. Assume that $\hat{\phi} \in H^{m+3 / 2}(\delta \Omega), \hat{\psi} \in H^{m+1 / 2}(\delta \Omega)$ are given. Furthermore, assume that $s \neq 0 .(\hat{u}, \hat{v}) \in H^{m+2}(\Omega) \times H^{m+2}\left(\Omega_{R}^{+}\right)$is a solution of (EP) if and only if $(\hat{u}, \hat{v})$ satisfies the system of integral equation (IE) given by

$$
\begin{aligned}
\hat{u} & =s \mathscr{V}^{i}[q \hat{u}]+\mathscr{D}^{i}[\hat{\phi}]-\mathscr{S}^{i}[\hat{\psi}]+k[\hat{u}, \hat{\phi}, \hat{\psi}, s], \\
\hat{v} & =s \mathscr{V}^{e}[q \hat{u}]+\mathscr{D}^{e}[\hat{\phi}]-\mathscr{S}^{e}[\hat{\psi}]+k[\hat{u}, \hat{\phi}, \hat{\psi}, s],
\end{aligned}
$$

where $k[\hat{u}, \hat{\phi}, \hat{\psi}, s]$ is the constant mapping given by

$$
k[\hat{u}, \hat{\phi}, \hat{\psi}, s]=A^{-1}\left(\int_{\Omega} q\left(\mathscr{S}^{i}[\hat{\psi}]-\mathscr{D}^{i}[\hat{\phi}]\right)-s \int_{\Omega} q \mathscr{V}^{i}[q \hat{u}]+s^{-1} \int_{\delta \Omega} \hat{\psi}\right),
$$

and $A=\int_{\Omega} q$. 
Proof. Assuming that $(\hat{u}, \hat{v})$ is a solution of (EP), then the compatibility condition (3.3) holds with $f \equiv s q \hat{u}$. Theorem 3.1 implies that there exists a constant $k$ depending on $s, \hat{u}, \hat{\phi}$, and $\hat{\psi}$ such that

$$
\begin{aligned}
& \hat{u}=s \mathscr{V}^{i}[q \hat{u}]+\mathscr{D}^{i}[\hat{\phi}]-\mathscr{S}^{i}[\hat{\psi}]+k, \\
& \hat{v}=s \mathscr{V}^{e}[q \hat{u}]+\mathscr{D}^{e}[\hat{\phi}]-\mathscr{S}^{e}[\hat{\psi}]+k .
\end{aligned}
$$

We multiply the first equation of (3.12) by $s q$, integrate over $\Omega$ and use the fact that $\int_{\Omega} s q \hat{u}=\int_{\delta \Omega} \hat{\psi}$ to get

$$
\int_{\Omega} s q \hat{u}=s \int_{\Omega} q\left(s \mathscr{V}^{i}[q \hat{u}]+\mathscr{D}^{i}[\hat{\phi}]-\mathscr{S}^{i}[\hat{\psi}]+k\right)=\int_{\delta \Omega} \hat{\psi} .
$$

Using the last equality to solve for $k$, we get that (3.11) holds for $s \neq 0$.

Next, assuming that $(\hat{u}, \hat{v})$ satisfies $(3.10)$, then the properties of the potentials and Lemma 3.1 immediately yield

$$
\Delta \hat{u}-s q \hat{u}=0, \quad \Delta \hat{v}=0, \quad \hat{u}^{-}=\hat{v}^{+}+\hat{\phi}, \quad \hat{u}_{n}^{-}=\hat{v}_{n}^{+}+\hat{\psi} .
$$

We multiply the first equation of (3.10) by $s q$ and integrate over $\Omega$ to get $\int_{\Omega} s q \hat{u}=$ $\int_{\delta \Omega} \hat{\psi}$, which we use in a similar argument as in the proof of Theorem 3.1 to show that $\hat{v}=O(1),|\nabla \hat{v}|=O\left(|x|^{-2}\right)$ as $|x| \rightarrow \infty$.

Our next step is to prove existence of solutions for (DEP). In view of Theorem 3.2 this is equivalent to finding solution to an integral equation system. Before we proceed, in order to simplify the notation, we will make the following definitions. We define the operators $V^{i}, D^{i}, S^{i}$ as follows:

$$
\begin{gathered}
V^{i}[f]=\mathscr{V}^{i}[q f]-A^{-1} \int_{\Omega} q \mathscr{V}^{i}[q f], \\
D^{i}[\varphi]=\mathscr{D} i[\varphi]-A^{-1} \int_{\Omega} q \mathscr{D}^{i}[\varphi], \\
S^{i}[\varphi]=\mathscr{S}^{i}[\varphi]-A^{-1} \int_{\Omega} q \mathscr{S}^{i}[\varphi] .
\end{gathered}
$$

We define the operators $V^{e}, S^{e}, D^{e}$ by changing the superscript $i$ to $e$ in the above definitions. Here $A$ is defined to be $A:=\int_{\Omega} q$.

With these definitions we can write problem (IE) as follows:

Problem (IE). Find $\hat{u}, \hat{v}$ such that

$$
\begin{aligned}
& \hat{u}=s V^{i}[q \hat{u}]+D^{i}[\hat{\phi}]-S^{i}[\hat{\psi}]+(A s)^{-1} \int_{\delta \Omega} \hat{\psi}, \\
& \hat{v}=s V^{e}[q \hat{u}]+D^{e}[\hat{\phi}]-S^{e}[\hat{\psi}]+(A s)^{-1} \int_{\delta \Omega} \hat{\psi} .
\end{aligned}
$$

THEOREM 3.3. If $\hat{\phi} \in H^{m+3 / 2}(\delta \Omega), \hat{\psi} \in H^{m+1 / 2}(\delta \Omega)$, then there exists a countable set $\Lambda=\left\{s_{k}\right\}_{k=1}^{\infty} \cup\{0\} \subset(-\infty, 0]$ such that if $s \in \mathbb{C} / \Lambda$ then (IE) has unique solution $(\hat{u}, \hat{v}) \in H^{m+2}(\Omega) \times H^{m+2}\left(\Omega_{R}^{+}\right)$. Moreover, the only accumulation point of $\Lambda$ is $-\infty$.

Proof. The main idea for the proof is to show uniqueness for $s \in \mathbb{C}(-\infty, 0]$ and then use Fredholm theory to prove existence. 
Assuming that $(\hat{u}, \hat{v})$ is a solution of (IE) for $(\hat{\phi}, \hat{\psi})=(0,0)$ and that $s \in$ $\mathbb{C} /(-\infty, 0]$, then by Theorem 3.2, $(\hat{u}, \hat{v})$ is a solution of (EP). We multiply the first equation of (3.1) by $\overline{\hat{u}}$ and the second equation by $\overline{\hat{v}}$. We integrate over $\Omega$ and $\Omega^{+}$respectively, using integration by parts and a limiting argument in $\Omega^{+}$to get

$$
\begin{gathered}
\int_{\Omega}|\nabla \hat{u}|^{2}+s \int_{\Omega} q|\hat{u}|^{2}=\int_{\delta \Omega} \hat{u}_{n}^{-}(\overline{\hat{u}})^{-}, \\
\int_{\Omega^{+}}|\nabla \hat{v}|^{2}=-\int_{\delta \Omega} \hat{v}_{n}^{+}(\overline{\hat{v}})^{+} .
\end{gathered}
$$

Adding (3.14) and (3.15) and using the transition relations across the boundary we get

$$
\int_{\Omega}|\nabla \hat{u}|^{2}+s \int_{\Omega} q|\hat{u}|^{2}+\int_{\Omega^{+}}|\nabla \hat{v}|^{2}=0
$$

If $\operatorname{Im}(s) \neq 0$, then $\int_{\Omega} q|\hat{u}|^{2}=0$, which implies that $\int_{\Omega^{+}}|\nabla \hat{v}|^{2}=0$. Hence $\hat{u} \equiv 0$ and $\hat{v} \equiv c$. The transition condition $\hat{u}^{-}=\hat{v}^{+}$then yields $\hat{v}^{+} \equiv 0 . \operatorname{If} \operatorname{Im}(s)=0$ and $\operatorname{Re}(s)>0$ then (3.16) again implies that $\int_{\Omega} q|\hat{u}|^{2}=0$ and that $\int_{\Omega^{+}}|\nabla \hat{v}|^{2}=0$ which as before shows that $\hat{u} \equiv \hat{v} \equiv 0$. Thus, the only homogeneous solution of (IE) for $s \in \mathbb{C} \in(-\infty, 0]$ is the zero solution.

Now, to finish the proof we only need to show that the operator $I-s V^{i}$ is invertible $\forall s \in \mathbb{C} / \Lambda$. We note that $V^{i}$ is a bounded operator from $H^{m}(\Omega)$ into $H^{m+2}(\Omega) \forall m \geq$ 0 . Hence, $V^{i}$ is a compact operator from $H^{m}(\Omega)$ into $H^{m}(\Omega)$. Since for $s \in$ $\mathbb{C} /(-\infty, 0]$ the only homogeneous solution of (IE) is the zero solution, we conclude that $\left(I-s V^{i}\right)$ has an inverse $\forall s \in \mathbb{C} /(-\infty, 0]$ and that the set of points for which the inverse of $\left(I-s V^{i}\right)$ fails to exist is a countable set $\left\{s_{k}\right\}_{k=1}^{\infty}$ having $-\infty$ as the only possible accumulation point.

4. A priori bounds. Our purpose here is to estimate the solution of (IE) for large values of $s$. This will enable us to take an inverse Laplace transform obtaining a solution to (PEP) and deriving its large time behavior. We start by making the following definitions.

Let $\delta$ be a positive number, we define $\Sigma_{\delta}$ to be the set of points in the complex plane:

$$
\Sigma_{\delta}=\{s \in \mathbb{C}:|\arg (s)| \leq \pi / 2+\delta\} \cap\{|s| \geq 1\} .
$$

Let $(\hat{u}[\hat{\phi}, \hat{\psi}], \hat{v}[\hat{\phi}, \hat{\psi}])$ denote the solution of $(\mathrm{IE})$. We define $\left(\hat{u}_{1}[\hat{\phi}], \hat{v}_{1}[\hat{\phi}]\right)$ and $\left(\hat{u}_{2}[\hat{\psi}], \hat{v}_{2}[\hat{\psi}]\right)$ to be

$$
\begin{aligned}
\left(\hat{u}_{1}[\hat{\phi}], \hat{v}_{1}[\hat{\phi}]\right) & :=(\hat{u}[\hat{\phi}, 0], \hat{v}[\hat{\phi}, 0]) \\
{\left[\hat{u}_{2}[\hat{\psi}], \hat{v}_{2}[\hat{\psi}]\right) } & :=(\hat{u}[0, \hat{\psi}], \hat{v}[0, \hat{\psi}]) .
\end{aligned}
$$

THEOREM 4.1. Assume that $\hat{\phi} \in H^{m+3 / 2}(\delta \Omega), \hat{\psi} \in H^{m+1 / 2}(\delta \Omega)$ and that $\theta_{0}$ with $0 \leq \theta_{0}<\pi / 2$ are given. There exists a positive real number $c_{1}\left(\Omega, m, \theta_{0}\right)$ such that $\forall s \in \Sigma_{\theta_{0}}$ we have
(i) $\|\hat{u}[\hat{\phi}, \hat{\psi}]\|_{m, \Omega} \leq c|s|^{m / 2}\left\{\|\hat{\phi}\|_{m+3 / 2, \delta \Omega}+\|\hat{\psi}\|_{m+1 / 2, \delta \Omega}\right\}$
(ii) $\|\hat{u}[\hat{\phi}, \hat{\psi}]\|_{m+1, \Omega} \leq c|s|^{m / 2+1 / 2}\left\{\|\hat{\phi}\|_{m+3 / 2, \delta \Omega}+\|\hat{\psi}\|_{m+1 / 2, \delta \Omega}\right\}$
(iii) $\|\hat{u}[\hat{\phi}, \hat{\psi}]\|_{m+2, \Omega} \leq c|s|^{m / 2+1}\left\{\|\hat{\phi}\|_{m+3 / 2, \delta \Omega}+\|\hat{\psi}\|_{m+1 / 2, \delta \Omega}\right\}$. 
Proof. We start with the case for which $m=0$ and $\hat{\psi}=0$. For $s \in \Sigma_{\theta_{0}}$, $\left(\hat{u}_{1}[\hat{\phi}], \hat{v}_{1}[\hat{\phi}]\right)$ is a solution of (EP). Using an argument similar to the one in the proof of Theorem 3.1 we get

$$
\int_{\Omega^{+}}\left|\nabla \hat{v}_{1}\right|^{2}+s \int_{\Omega} q\left|\hat{u}_{1}\right|^{2}+\int_{\Omega}\left|\nabla \hat{u}_{1}\right|^{2}=\int_{\delta \Omega}\left(\partial_{n} \hat{v}_{1}\right)^{+} \overline{\hat{\phi}} .
$$

We know that $\hat{v}_{1}=s V^{e}\left[\hat{u}_{1}\right]+D^{e}[\hat{\phi}]$, from which we get

$$
\left(\partial_{n} \hat{v}_{1}\right)^{+}=s\left(\partial_{n} V^{e}\left[\hat{u}_{1}\right]\right)^{+}+\left(\partial_{n} D^{e}[\hat{\phi}]\right)^{+} .
$$

Using Lemma 3.1 we rewrite (4.2) as

$$
\left(\partial_{n} \hat{v}_{1}\right)^{+}=s\left(\partial_{n} V^{i}\left[\hat{u}_{1}\right]\right)^{-}+\left(\partial_{n} D^{i}[\hat{\phi}]\right)^{-} .
$$

Using (4.3) trace Theorems and the fact that $V^{i} \in B\left[H^{m}(\Omega): H^{m+2}(\Omega)\right]$ and that $D^{i} \in B\left[H^{m+3 / 2}(\delta \Omega): H^{m+2}(\Omega)\right]$ we get

$$
\begin{aligned}
\left\|\left(\partial_{n} \hat{v}_{1}\right)^{+}\right\|_{1 / 2, \delta \Omega} & \leq c(\Omega)\left(|s|\left\|V^{i}\left[\hat{u}_{1}\right]\right\|_{2, \Omega}+\left\|D^{i}[\hat{\phi}]\right\|_{2, \Omega}\right) \\
& \leq c(\Omega) \max \left\{\left\|V^{i}\right\|_{0},\left\|D^{i}\right\|_{0}\right\}\left(|s|\left\|\hat{u}_{1}\right\|_{0, \Omega}+\|\hat{\phi}\|_{3 / 2, \delta \Omega}\right) .
\end{aligned}
$$

Taking the absolute value of (4.1) and estimating the right-hand side using (4.4) we get

$$
\left.\left|\int_{\Omega^{+}}\right| \nabla \hat{v}_{1}\right|^{2}+s \int_{\Omega} q\left|\hat{u}_{1}\right|^{2}+\int_{\Omega}\left|\nabla \hat{u}_{1}\right|^{2} \mid \leq c(\Omega)\left(|s|\left\|\hat{u}_{1}\right\|_{0, \Omega}+\|\hat{\phi}\|_{3 / 2, \delta \Omega}\right)\|\hat{\phi}\|_{3 / 2, \delta \Omega} .
$$

Now, for $s=\sigma+i \eta$ with $\sigma>0$, we use (4.5) to obtain

$$
\begin{aligned}
\min _{x \in \bar{\Omega}}\{q(x)\}|s| \int_{\Omega}\left|\hat{u}_{1}\right|^{2} & \leq|s| \int_{\Omega} q\left|\hat{u}_{1}\right|^{2} \\
& \leq\left.\left|\int_{\Omega^{+}}\right| \nabla \hat{v}_{1}\right|^{2}+s \int_{\Omega} q\left|\hat{u}_{1}\right|^{2}+\int_{\Omega}\left|\nabla \hat{u}_{1}\right|^{2} \mid \\
& \leq c(\Omega)\left(|s|\left\|\hat{u}_{1}\right\|_{0, \Omega}+\|\hat{\phi}\|_{3 / 2, \delta \Omega}\right)\|\hat{\phi}\|_{3 / 2, \delta \Omega} \\
& \leq c(\Omega) \frac{\varepsilon}{2}|s|\left\|\hat{u}_{1}\right\|_{0, \Omega}^{2}+c(\Omega)\left(\frac{|s|}{2 \varepsilon}+1\right)\|\hat{\phi}\|_{3 / 2, \delta \Omega}^{2} .
\end{aligned}
$$

With the help of (4.6) we get

$$
\left(\underline{\alpha}-c(\Omega) \frac{\varepsilon}{2}\right)|s|\left\|\hat{u}_{1}\right\|_{0, \Omega}^{2} \leq c\left(\frac{|s|}{2 \varepsilon}+1\right)\|\hat{\phi}\|_{3 / 2, \delta \Omega}^{2},
$$

where $\underline{\alpha}:=\min _{x \in \bar{\Omega}}\{q(x)\}$. Since $\underline{\alpha}>0$, we choose $\varepsilon$ so small that $(\underline{\alpha}-c(\Omega) \varepsilon / 2)>$ 0 , and obtain

$$
\left\|\hat{u}_{1}\right\|_{0, \Omega}^{2} \leq c\left(1+|s|^{-1}\right)\|\hat{\phi}\|_{3 / 2, \delta \Omega}^{2} .
$$

For $|s| \geq 1$ and $\operatorname{Re}(s)>0$ we get

$$
\left\|\hat{u}_{1}\right\|_{0, \Omega}^{2} \leq c(\Omega)\|\hat{\phi}\|_{3 / 2, \delta \Omega}^{2} .
$$


With the help of (4.9) we estimate $\int_{\Omega}\left|\nabla \hat{u}_{1}\right|^{2}$ for $s=\sigma+i \eta$ with $\sigma>0$.

$$
\begin{aligned}
\int_{\Omega}\left|\nabla \hat{u}_{1}\right|^{2} & \leq\left.\left|\int_{\Omega^{+}}\right| \nabla \hat{v}_{1}\right|^{2}+s \int_{\Omega} q\left|\hat{u}_{1}\right|^{2}+\int_{\Omega}\left|\nabla \hat{u}_{1}\right|^{2} \mid \\
& \leq c(\Omega)\left(|s|\left\|\hat{u}_{1}\right\|_{0, \Omega}+\|\hat{\phi}\|_{3 / 2, \delta \Omega}\right)\|\hat{\phi}\|_{3 / 2, \delta \Omega} \\
& \leq c|s|\|\hat{\phi}\|_{3 / 2, \delta \Omega .}^{2}
\end{aligned}
$$

Combining (4.9) and (4.10) we obtain

$$
\left\|\hat{u}_{1}\right\|_{1, \Omega} \leq c|s|^{1 / 2}\|\hat{\phi}\|_{3 / 2, \delta \Omega}, \quad \operatorname{Re}(s)>0, \quad|s| \geq 1 .
$$

We need to get similar estimates for $\hat{u}_{1}$ in

$$
\Pi_{\theta_{0}}:=\left\{s \in \mathbb{C}:|s| \geq 1, \frac{\pi}{2} \leq|\arg (s)| \leq \frac{\pi}{2}+\theta_{0}\right\} .
$$

If $s=\sigma+i \eta, s \in \Pi_{\theta_{0}}$ then it is easy to check that

$$
|s| \cos \theta_{0} \leq|\eta|, \quad 0 \leq-\sigma \leq|s| \sin \theta_{0} .
$$

By a little modification of the argument leading to (4.9) and (4.11) and the use of (4.12) we derive the following estimates

$$
\begin{gathered}
\left\|\hat{u}_{1}\right\|_{0, \Omega} \leq c\|\hat{\phi}\|_{3 / 2, \delta \Omega}, \quad \text { for }|s| \in \Pi_{\theta_{0}} \\
\left\|\hat{u}_{1}\right\|_{1, \Omega} \leq c|s|^{1 / 2}\|\hat{\phi}\|_{3 / 2, \delta \Omega}, \quad \text { for }|s| \in \Pi_{\theta_{0}} .
\end{gathered}
$$

To get estimates for $\hat{u}_{1}$ in the $H^{2}(\Omega)$-norm $(m=0)$, we just use the fact that $\hat{u}_{1}$ satisfies

$$
\hat{u}_{1}=s V^{i}\left[\hat{u}_{1}\right]+D^{i}[\hat{\phi}] .
$$

For $s \in \Pi_{\theta_{0}}$, taking norms in (4.15) and using the fact that $V^{i} \in B\left[H^{m}(\Omega): H^{m+2}(\Omega)\right]$ and $D^{i} \in B\left[H^{m+3 / 2}(\delta \Omega): H^{m+2}(\Omega)\right]$ we get

$$
\begin{aligned}
\left\|\hat{u}_{1}\right\|_{2, \Omega} & \leq|s|\left\|V^{i}\left[\hat{u}_{1}\right]\right\|_{2, \Omega}+\left\|D^{i}[\hat{\phi}]\right\|_{2, \Omega} \\
& \leq|s|\left\|V^{i}\right\|_{0}\left\|\hat{u}_{1}\right\|_{0, \Omega}+\left\|D^{i}\right\|_{0}\|\hat{\phi}\|_{3 / 2, \delta \Omega} \\
& \leq c(|s|+1)\|\hat{\phi}\|_{3 / 2, \delta \Omega} \\
& \leq c|s|\|\hat{\phi}\|_{3 / 2, \delta \Omega} .
\end{aligned}
$$

An induction argument over $m$ using (4.15) and a calculation similar to the one leading to (4.16) proves the Theorem for the case $\hat{\psi} \equiv 0$. A slight modification of the previous arguments proves the Theorem for the case $\hat{\phi} \equiv 0$.

5. Proofs of Theorem 2.1 and Theorem 2.2. Let $X$ be a separable Hilbert space and $\varphi(t)$ be a function from $[0, \infty)$ into $\mathbf{X}$. If $\varphi \in C[[0, \infty): \mathbf{X}]$ and if $\int_{0}^{\infty}\|\varphi(t)\|_{x} e^{-\rho_{0} t} d t<\infty$, then we can take the Laplace transform of $\varphi$ denoted by $\hat{\varphi}$ and defined by

$$
\hat{\varphi}(s)=\int_{0}^{\infty} \varphi(t) e^{-s t} d t, \quad \operatorname{Re}(s) \geq \rho_{0} .
$$


We have

$$
\varphi(t)=(2 \pi)^{-1} \int_{L\left(\delta_{0}\right)} \hat{\varphi}(s) e^{s t} d s,
$$

where $L\left(\delta_{0}\right)=\left\{s \in \mathbb{C}: s=\delta_{0}+\mathfrak{i} \eta,-\infty<\eta<\infty, \delta_{0} \geq \rho_{0}\right\}$. It is easy to check that if $\varphi \in Z^{m}\left[\mathbf{X}, \rho_{0}\right]$, then $\|\hat{\varphi}\|_{x} \leq\left(\int_{0}^{\infty}\left\|\varphi^{(m)}(t)\right\|_{x} e^{-\rho_{0} t} d t\right)|s|^{-m}$.

We introduce the following notation. Let $f$ be a smooth function on a region $G \in \mathbb{R}^{2}$, we define $\||f|\|_{n, G}$ to be $\||f|\|_{n, G}=\sum_{|\alpha| \leq n}\left\|D^{\alpha} f\right\|_{\infty}$.

Proposition 5.1. There exists a countable set $\varpi=\left\{s_{k}\right\}_{k=1}^{\infty} \subset\left(-\infty,-\left\|V^{i}\right\|_{m}^{-1}\right]$ such that if $s \in \mathbb{C} / \varpi$ then the mappings

$$
s \rightarrow\left(I-V^{i}\right)^{-1} D^{i}, \quad s \rightarrow\left(I-V^{i}\right)^{-1} S^{i}
$$

are analytic mappings from $\mathbb{C} / \varpi$ into $B\left[H^{m+3 / 2}(\delta \Omega), H^{m+2}(\Omega)\right]$ and into $B\left[H^{m+1 / 2}(\delta \Omega), H^{m+2}(\Omega)\right]$, respectively.

Proof. From the proof of Theorem 3.3 we have that $\left(I-V^{i}\right)^{-1}$ exists except on a countable set $\left\{s_{k}\right\}_{k=1}^{\infty} \subset(-\infty, 0]$. Moreover, if $|s|<\left\|V^{i}\right\|_{m}^{-1}$, standard successive approximations yield the existence of $\left(I-V^{i}\right)^{-1}$. Hence, $\left(I-V^{i}\right)^{-1}$ exists except on a countable set $\varpi=\left\{s_{k}\right\}_{k=1}^{\infty} \subset\left(-\infty,-\left\|V^{i}\right\|_{m}^{-1}\right]$. Analyticity follows immediately from the fact that $\left(I-V^{i}\right)^{-1}$ exists for $s \in \mathbb{C} / \varpi$ and the facts that $S^{i}, D^{i}$ are in $B\left[H^{m+1 / 2}(\delta \Omega), H^{m+2}(\Omega)\right]$ and $B\left[H^{m+3 / 2}(\delta \Omega), H^{m+2}(\Omega)\right]$, respectively.

Proof of Theorem 2.1. Let $\hat{\phi}, \hat{\psi}$ be the Laplace transform of $\phi, \psi$ and let $\delta_{0}:=\max \left(\left|\rho_{0}\right|,\left|\rho_{1}\right|\right)$. We define $u, v$ to be

$$
\begin{aligned}
& u:=(2 \pi)^{-1} \int_{L\left(\delta_{0}\right)} \hat{u}[\hat{\phi}, \hat{\psi}](s) e^{s t} d s, \\
& v:=(2 \pi)^{-1} \int_{L\left(\delta_{0}\right)} \hat{v}[\hat{\phi}, \hat{\psi}](s) e^{s t} d s .
\end{aligned}
$$

Since $\phi \in Z^{I[m / 2]+3}\left[H^{m+3 / 2}(\delta \Omega), \rho_{0}\right]$ and $\psi \in Z^{I[m / 2]+3}\left[H^{m+1 / 2}(\delta \Omega), \rho_{1}\right]$ we have

$$
\begin{array}{ll}
\|\hat{\phi}\|_{m+3 / 2, \delta \Omega} \leq c_{1}|s|^{-(I[m / 2]+3)} & \operatorname{Re}(s)>\rho_{0}, \\
\|\hat{\psi}\|_{m+1 / 2, \delta \Omega} \leq c_{2}|s|^{-(I[m / 2]+3)} & \operatorname{Re}(s)>\rho_{0},
\end{array}
$$

where $c_{1}, c_{2}$ are independent of $s$.

In view of Theorem 4.1 and Proposition 5.1 we get that

$$
\begin{aligned}
& \|\hat{u}[\hat{\phi}, \hat{\psi}]\|_{m, \Omega} \leq c|s|^{-3} \text { for } \operatorname{Re}(s) \geq \delta_{0}, \\
& \|\hat{u}[\hat{\phi}, \hat{\psi}]\|_{m+2, \Omega} \leq c|s|^{-2} \text { for } \operatorname{Re}(s) \geq \delta_{0} .
\end{aligned}
$$

Thus the integral (5.1) converges absolutely and is well defined. Moreover, since $\hat{v}[\hat{\phi}, \hat{\psi}]$ is a solution of (IE) satisfying

$$
\hat{v}[\hat{\phi}, \hat{\psi}]=s V^{e}[\hat{u}]+D^{e}[\hat{\phi}]-S^{\mathcal{C}}[\hat{\psi}]+(A s)^{-1} \int_{\delta \Omega} \hat{\psi},
$$


and since

$$
\begin{gathered}
V^{e} \in B\left[H^{m}(\Omega), H^{m+2}\left(\Omega_{R}^{+}\right)\right] \cap B\left[L^{2}(\Omega), C^{n}\left(\Omega_{R}^{+}(\delta)\right)\right], \\
S^{e} \in B\left[H^{m+1 / 2}(\delta \Omega), H^{m+2}\left(\Omega_{R}^{+}\right)\right] \cap B\left[L^{2}(\delta \Omega), C^{n}\left(\Omega_{R}^{+}(\delta)\right)\right], \\
\left.D^{e} \in B\left[H^{m+3 / 2}(\delta \Omega), H^{m+2}\left(\Omega_{R}^{+}\right)\right)\right] \cap B\left[L^{2}(\delta \Omega), C^{n}\left(\Omega_{R}^{+}(\delta)\right)\right],
\end{gathered}
$$

we can use (5.7), (5.6), and (5.5) to get

$$
\begin{gathered}
\|\hat{v}[\hat{\phi}, \hat{\psi}]\|_{m+2, \Omega_{R}^{+}} \leq c\left(\Omega_{R}^{+}\right)|s|^{-2} \text { for } \operatorname{Re}(s) \geq \delta_{0}, \\
\|\hat{v}[\hat{\phi}, \hat{\psi}]\| \|_{n, \Omega_{R}^{+}(\delta)} \leq c\left(\Omega_{R}^{+}(\delta)\right)|s|^{-2} \text { for } \operatorname{Re}(s) \geq \delta_{0} .
\end{gathered}
$$

Hence the integral (5.2) is well defined pointwise, and also as an integral of a function of the variable $s$ taking value in $H^{m}\left(\Omega_{R}^{+}\right)$.

From (5.5) and (5.6) it follows that $u \in C\left[[0, \infty): H^{m+2}(\Omega)\right] \cap C^{1}\left[[0, \infty): H^{m}(\Omega)\right]$ and that

$$
\frac{d}{d t} u=(2 \pi)^{-1} \int_{L\left(\delta_{0}\right)} s \hat{u}[\hat{\phi}, \hat{\psi}](s) e^{s t} d s .
$$

Making use of Theorem 3.2, Eq. (5.10) and the fact that the Laplacian is a bounded operator from $H^{m+2}(\Omega)$ into $H^{m}(\Omega)$ we get

$$
\frac{d}{d t} u-\Delta u=(2 \pi)^{-1} \int_{L\left(\delta_{0}\right)}(s q \hat{u}-\Delta \hat{u}) e^{s t} d s=0 .
$$

From (5.8), (5.9), and Theorem 3.2, we get that $v \in C\left[[0, \infty): H^{2}\left(\Omega_{R}^{+}\right)\right] \cap$ $C\left[[0, \infty): C^{1}\left(\Omega_{R}^{+}(\delta)\right)\right]$ and that

$$
\Delta v=(2 \pi)^{-1} \int_{L\left(\delta_{0}\right)} \Delta \hat{v}[\hat{\phi}, \hat{\psi}](s) e^{s t} d s=0 .
$$

Using the boundedness of the trace operators we get

$$
\begin{aligned}
u^{-}-v^{+} & =(2 \pi)^{-1}\left(\int_{L\left(\delta_{0}\right)} \hat{u} e^{s t}\right)^{-}-(2 \pi)^{-1}\left(\int_{L\left(\delta_{0}\right)} \hat{v} e^{s t}\right)^{+} \\
& =(2 \pi)^{-1} \int_{L\left(\delta_{0}\right)}\left(\hat{u}^{-}-\hat{v}^{+}\right) e^{s t} d s \\
& =(2 \pi)^{-1} \int_{L\left(\delta_{0}\right)} \hat{\phi} e^{s t} d s \\
& =\phi(t) .
\end{aligned}
$$

A similar argument yields: $u_{n}^{-}-v_{n}^{+}=\psi$.

For $x$ sufficiently large, we use Lemma 3.2, (5.5), and (5.6) to get

$$
\begin{array}{cc}
|\hat{v}[\hat{\phi}, \hat{\psi}]| \leq c|s|^{-2} & \operatorname{Re}(s) \geq \delta_{0}, \\
|\hat{v}[\hat{\phi}, \hat{\psi}]| \leq c|x|^{-2}|s|^{-2} & \operatorname{Re}(s) \geq \delta_{0},
\end{array}
$$


where $c$ is a constant independent of $x$. We estimate $v(x, t)$ using (5.12). We obtain

$$
\begin{aligned}
|v(x, t)| & =\left|(2 \pi)^{-1} \int_{L\left(\delta_{0}\right)} \hat{v}(s) e^{s t} d s\right| \\
& \leq c \int_{-\infty}^{\infty} \frac{e^{\delta_{0} t}}{\delta_{0}^{2}+\eta^{2}} d \eta \\
& \leq c\left(\delta_{0}, t\right) .
\end{aligned}
$$

Hence $v(x, t)=O(1)$ as $|x| \rightarrow \infty$. A similar argument shows that $\nabla v=O\left(|x|^{-2}\right)$ as $|x| \rightarrow \infty$.

For $\hat{u}$ in $H^{m+2}(\Omega)$, Eq. (5.6) states that $\hat{u}(s)=O\left(|s|^{-2}\right.$ ) for large $s$. Thus the integral (5.1) is well defined for $t \in(-\infty,+\infty)$ and since the integral is absolutely continuous, it defines a continuous function for $t \in(-\infty,+\infty)$. This, and a standard argument from Laplace transform theory imply that $u(0)=0$. The fact that $u(0)=0$ together with the fact that $u(t)$ is continuous in $H^{m+2}(\Omega)$ imply that $\lim _{t \downarrow 0} u=0$.

To show uniqueness, we assume that $(u, v)$ is a solution of $(\mathrm{PEP})$ for $(\phi, \psi)=$ $(0,0)$. A simple energy argument using the equations for (PEP) yields

$$
\int_{\Omega} \frac{1}{2} u^{2}(x, t) d x+\int_{0}^{t} \int_{\Omega}|\nabla u|^{2}+\int_{0}^{t} \int_{\Omega^{+}}|\nabla v|^{2} d x d t=0 .
$$

Since $q>0,(5.14)$ implies that $u(x, t) \equiv 0$. Moreover, since $v^{+}=u^{-}$we have that $v^{+}=0$. This, the fact that $\Delta v=0$, and the growth conditions for large $x$ imply that $v \equiv 0$.

Proof of Theorem 2.2. We consider the function $u-(A)^{-1} \int_{0}^{t} \int_{\delta \Omega} \psi$. In view of (5.1) this can be written as

$$
\begin{aligned}
u-(A)^{-1} \int_{0}^{t} \int_{\delta \Omega} \psi= & (2 \pi)^{-1} \int_{L\left(\delta_{0}\right)}\left(\hat{u}[\hat{\phi}, \hat{\psi}](s)-(A s)^{-1} \int_{\delta \Omega} \hat{\psi}\right) e^{s t} d s, \\
= & (2 \pi)^{-1} \int_{L\left(\delta_{0}\right)} \hat{u}_{1}[\hat{\phi}] e^{s t} d s \\
& +(2 \pi)^{-1} \int_{L\left(\delta_{0}\right)}\left(\hat{u}_{2}[\hat{\psi}]-(A s)^{-1} \int_{\delta \Omega} \hat{\psi}\right) e^{s t} d s .
\end{aligned}
$$

Let $I_{1}, I_{2}$ denote the first and second integral, respectively. The integrand of $I_{2}$ is given by

$$
\widehat{G}_{2}(s)=-\left(I-s V^{i}\right)^{-1}\left(S^{i}[\hat{\psi}]-(A s)^{-1} \int_{\delta \Omega} \hat{\psi}\right)-(A s)^{-1} \int_{\delta \Omega} \hat{\psi} .
$$

Using the identity

$$
\left(I-s V^{i}\right)^{-1}=I+s\left(I-s V^{i}\right)^{-1} V^{-1},
$$

we rewrite $(5.17)$ as

$$
\widehat{G}_{2}(s)=-\left(I-s V^{i}\right)^{-1} S^{i}[\hat{\psi}]+\left((A s)^{-1} \int_{\delta \Omega} \hat{\psi}\right)\left(I-s V^{i}\right)^{-1} V^{-1}[e],
$$

where $e(x) \equiv 1 \quad \forall x \in \Omega$. 
By Proposition 5.1 we have that $\widehat{G}_{2}(s)$ is analytic from $\mathbb{C} /\left(-\infty,-\left\|V^{i}\right\|_{m}^{-1}\right]$ into $H^{m+2}(\Omega)$. Using Theorem 4.1 and the analyticity of $\widehat{G}_{2}(s)$ we get that $\exists c$ independent of $s$ such that

$$
\left\|\widehat{G}_{2}(s)\right\|_{m+2, \Omega} \leq c|s|^{-2}, \quad \operatorname{Re}(s)>\max \left\{\rho_{1},-\left\|V^{i}\right\|_{m}^{-1} \| .\right.
$$

Hence by Cauchy Theorem we can shift the contour $L\left(\delta_{0}\right)$ to a straight line $L\left(\delta_{1}\right)$ lying in the left half of the complex plane with $0>\delta_{1}>\max \left\{\rho_{1},-\left\|V^{i}\right\|_{m}^{-1}\right\}$, and we have

$$
\begin{aligned}
\left\|I_{2}\right\|_{m+2, \Omega} & =\left\|(2 \pi)^{-1} \int_{L\left(\delta_{0}\right)} \widehat{G}_{2}(s)(s) e^{s t} d s\right\|_{m+2, \Omega} \\
& =\left\|(2 \pi)^{-1} \int_{L\left(\delta_{1}\right)} \widehat{G}_{2}(s) e^{s t} d s\right\|_{m+2, \Omega} \\
& \leq c e^{\delta_{1} t} .
\end{aligned}
$$

A similar argument for $I_{1}$ yields

$$
\left\|I_{1}\right\|_{m+2, \Omega} \leq c e^{\delta_{2} t}, \quad 0>\delta_{2}>\max \left\{\rho_{0},-\left\|V^{i}\right\|_{m}^{-1}\right\} .
$$

From (5.20) and (5.21) we get part (i) of Theorem 2.2. Part (ii) is done in a similar fashion.

6. The physical problem. We consider a uniform metallic cylinder of cross section $\Omega$ and parallel to the $x_{3}$-axis. The incident field is a transverse magnetic field in $\Omega^{+} \times \mathbb{R}$ of the form

$$
\begin{gathered}
\mathbf{E}_{e}^{0}=E^{0}\left(x_{1}, x_{2}\right) \mathbf{k}, \\
\mathbf{B}_{e}^{0}=B_{e 1}^{0}\left(x_{1}, x_{2}\right) \mathbf{i}+B_{e 2}^{0}\left(x_{1}, x_{2}\right) \mathbf{j} .
\end{gathered}
$$

We take the constitutive relation in the metal to be

$$
\mathbf{J}_{m}=\sigma \mathbf{E}_{m}, \quad \mathbf{B}_{m}=\mu_{0} \mathbf{H}_{m},
$$

and assume the exterior to be the air or a dielectric with constitutive relation of the form

$$
\mathbf{D}_{a}=\varepsilon_{0} \mathbf{E}_{a}, \quad \mathbf{B}_{a}=\mu_{0} \mathbf{H}_{a} .
$$

Letting $\left(\mathbf{E}_{e}, \mathbf{B}_{e}\right)$ denote the scattered field and $\left(\mathbf{E}_{m}, \mathbf{B}_{m}\right)$ denote the field in the metal, we write Maxwell's equations neglecting conduction current in the dielectric and displacement current in the metal. We get

in the dielectric

$$
\begin{aligned}
& \nabla \times \mathbf{E}_{e}=-\frac{\partial}{\partial t} \mathbf{B}_{e}, \quad \nabla \times \mathbf{B}_{e}=\varepsilon_{0} \mu_{0} \frac{\partial}{\partial t} \mathbf{E}_{e}, \\
& \nabla \cdot \mathbf{E}_{e}=0, \quad \nabla \cdot \mathbf{B}_{e}=0
\end{aligned}
$$

in the metal

$$
\begin{aligned}
\nabla \times \mathbf{E}_{m}=-\frac{\partial}{\partial t} \mathbf{B}_{m}, & \nabla \times \mathbf{B}_{m}=\mu_{0} \sigma \mathbf{E}_{m}, \\
\nabla \cdot \mathbf{E}_{m}=0, & \nabla \cdot \mathbf{B}_{m}=0 .
\end{aligned}
$$


Across the air-metal interface we have continuity of the tangential components of the electric and magnetic fields:

$$
\begin{aligned}
& \left(\mathbf{E}_{m}\right)_{\text {tang }}^{-}=\left(\mathbf{E}_{e}\right)_{\text {tang }}^{+}+\left(\mathbf{E}_{e}^{0}\right)_{\text {tang }}^{+}, \\
& \mu_{0}\left(\mathbf{B}_{m}\right)_{\text {tang }}^{-}=\mu_{0}\left(\left(\mathbf{B}_{e}\right)_{\text {tang }}^{+}+\left(\mathbf{B}_{e}^{0}\right)_{\text {tang }}^{+}\right) .
\end{aligned}
$$

Since the magnetic displacement is divergence-free we rewrite (6.5) and (6.6) in terms of the magnetic potential $u$ and get

$$
\begin{aligned}
& \mu_{0} \sigma \frac{\partial}{\partial t} u=\Delta u \text { in } \Omega \times(0, \infty), \quad \varepsilon_{0} \mu_{0} \frac{\partial^{2}}{\partial t^{2}} u=\Delta u \quad \text { in } \Omega^{+} \times(0, \infty) \\
& u^{-}=u^{+}+\left(u^{0}\right)^{+} \text {on } \partial \Omega \times(0, \infty), \quad u_{n}^{-}=u_{n}^{+}+\left(u_{n}^{0}\right)^{+} \text {on } \delta \Omega \times(0, \infty)
\end{aligned}
$$

A dimensional analysis in [4] shows that under slowly varying field we get the limit problem

$$
\begin{aligned}
& \mu_{0} \sigma \frac{\partial}{\partial t} u=\Delta u \text { in } \Omega \times(0, \infty), \quad \Delta u=0 \quad \text { in } \Omega^{+} \times(0, \infty) \\
& u^{-}=u^{+}+\left(u^{0}\right)^{+} \text {on } \delta \Omega \times(0, \infty), \quad u_{n}^{-}=u_{n}^{+}+\left(u_{n}^{0}\right)^{+} \text {on } \delta \Omega \times(0, \infty)
\end{aligned}
$$

with

$$
\begin{gathered}
u(x, t)-c(t) \log |x|=O(1) \quad \text { as }|x| \rightarrow \infty, \\
\nabla(u(x, t)-c(t) \log |x|)=O\left(|x|^{2}\right) \quad \text { as }|x| \rightarrow \infty,
\end{gathered}
$$

where $\left(u^{0}\right)^{+},\left(u_{n}^{0}\right)^{+}$, and $c(t)$ are given functions.

If we choose a point $x_{0} \in \Omega$ and define the function $v$ on $\Omega^{+} \times(0, \infty)$ by

$$
v=u(x, t)-c(t) \log \left|x-x_{0}\right| \quad \forall x \in \Omega^{+} \times(0, \infty),
$$

we obtain Problem (PEP).

For low frequency, Problem (PEP) gives a good description of the physical phenomenon near and inside the obstacle. However, for large distances from the obstacle, this model is not expected to be accurate.

\section{REFERENCES}

[1] S. i. Hariharan and R. C. MacCamy, Integral equation procedures for eddy current problems, J. Comp. Phys. 45, 80-89 (1982)

[2] S. I. Hariharan and R. C. MacCamy, Low frequency acoustic and electromagnetic scattering, Appl. Numer. Math. 2, 29-35 (1986)

[3] A. Aldroubi, A two-dimensional eddy current problem, Thesis, Dept. of Mathematics, CarnegieMiellon, 1987

[4] R. C. MacCamy and M. Suri, A time dependent interface problem for two dimensional eddy currents, Quart. Appl. Math. 46, 675-690 (1987) 